\title{
Mesoscopic fluctuations in the shot-noise power of metals
}

\author{
M. J. M. de Jong \\ Philips Research Laboratories, 5600 JA Eindhoven, The Netherlands \\ C. W. J. Beenakker \\ Instituut-Lorentz, University of Leiden, P.O. Box 9506, 2300 RA Leiden, The Netherlands
}

(Received 24 July 1992)

\begin{abstract}
The sample-to-sample fluctuations in the shot-noise power of a quasi-one-dimensional, phasecoherent, metallic, diffusive conductor are studied by extending the random-matrix theory of universal conductance fluctuations. The variance of the shot-noise power is shown to be independent of the sample size and the degree of disorder. The precise numerical value is calculated. Furthermore, a weak-localization effect in the average shot-noise power is found. The effect of inelastic scattering for conductors longer than the phase-coherence length is discussed.
\end{abstract}

\section{INTRODUCTION}

Recently, the classical problem of shot noise has been reinvestigated for quantum systems. ${ }^{1}$ Shot noise is the time-dependent fluctuation in the electrical current due to the discreteness of the charge of the carriers. It has been found that the shot-noise power $P$ is suppressed below the classical value of a Poisson process ${ }^{2}$ ( $P_{\text {Poisson }}=2 e \bar{I}$, with $\bar{I}$ the time-averaged current) as a consequence of noiseless open quantum channels. In particular, it was shown by Büttiker and one of the authors ${ }^{3}$ that the average noise power $\langle P\rangle$ in the diffusive transport regime is one-third of the Poisson value. The "average" here refers to an average over an ensemble of impurity configurations. It is well known in mesoscopic physics that transport properties may have large fluctuations around the average from sample to sample. ${ }^{4}$ Such "mesoscopic fluctuations" in the conductance were shown ${ }^{5,6}$ to have the root-mean-square value $e^{2} / h$ times a coefficient of order unity, independent of the size of the sample and the degree of disorder. Hence the name "universal conductance fluctuations" (UCF). In Ref. 3 it was argued on general grounds that the shot-noise power has mesoscopic fluctuations of order $\left(e^{2} / h\right) e|V|$, with $V$ the applied voltage. The purpose of the present paper is to give an explicit calculation of the root-mean-square value of the shot-noise power, $\operatorname{rms} P$, of disordered conductors, much longer than wide, but shorter than the localization length. It will be shown that, in the case of phase-coherent transport, these fluctuations are universal in the same sense as UCF and the precise numerical value will be calculated.

The starting point is the shot-noise formula derived by Büttiker. ${ }^{7}$ It expresses the zero-temperature, zerofrequency shot-noise power $P$ of a spin-degenerate twoprobe conductor over which a small voltage $V$ is applied, entirely in terms of transmission matrices $\underline{t}$ at the Fermi energy:

$$
\begin{aligned}
P & =2 e|V| \frac{2 e^{2}}{h} \operatorname{Tr}\left[\underline{t} \underline{t}^{\dagger}\left(\underline{1}-\underline{t} \underline{t}^{\dagger}\right)\right] \\
& =2 e|V| \frac{2 e^{2}}{h} \sum_{n=1}^{N} \mathcal{T}_{n}\left(1-\mathcal{T}_{n}\right),
\end{aligned}
$$

where $\mathcal{T}_{n}$ denotes an eigenvalue of $\underline{t} \underline{t}^{\dagger}$ and $N$ is the number of channels. Equation (1) is the multichannel generalization of the single-channel formulas found earlier. ${ }^{8-10}$ Using the Landauer formula

$$
G=\frac{2 e^{2}}{h} \operatorname{Tr} \underline{t} \underline{t}^{\dagger}=\frac{2 e^{2}}{h} \sum_{n=1}^{N} \mathcal{T}_{n}
$$

for the conductance $G=\bar{I} / V$, one finds from Eq. (1) that $P=P_{\text {Poisson }}$ if all transmission eigenvalues are small $\left(\mathcal{I}_{n} \ll 1\right.$, for all $\left.n\right)$. In a phase-coherent conductor, however, the $\mathcal{T}_{n}$ 's are either exponentially small (closed channels) or of order unity (open channels). ${ }^{11}$ This leads to sub-Poissonian shot noise when the ensemble average is taken. ${ }^{3}$

To determine the fluctuations in $P$ around $\langle P\rangle$ one can, in principle, use a diagrammatic Green's function method, as in the original theories of UCF. ${ }^{5,6}$ In this paper, however, the equivalent random-matrix method ${ }^{11-16}$ will be used, as it makes contact naturally with Eq. (1), where the shot-noise power is expressed as a function of random transmission matrices. The central quantity in the random-matrix theory of quantum transport is the distribution $w\left(\left\{\lambda_{1}, \lambda_{2}, \ldots, \lambda_{N}\right\}\right)$ of eigenparameters $\lambda_{n} \in[0, \infty)$, related to the transmission eigenvalues by $\mathcal{T}_{n} \equiv\left(1+\lambda_{n}\right)^{-1}$. The so-called local approach, which is based on the properties of small segments of the conductor, leads to a diffusion equation for the evolution of this distribution with length $L .^{13-15}$ The diffusion equation depends on the symmetry properties of the randommatrix ensemble. It can be written in a unified way using the symmetry parameter $\beta$, where $\beta=1$ in the presence and $\beta=2$ in the absence of time-reversal symmetry. 
For a sample with $N$ channels, a length $L$ and an elastic mean free path $\ell$, with the definition $s \equiv L / \ell$, the diffusion equation is given by ${ }^{13,15}$

$$
\begin{aligned}
\frac{\partial}{\partial s} w_{s}^{(\beta)}\left(\left\{\lambda_{2}\right\}\right)=\frac{2}{\beta N+2-\beta} & \\
\times \sum_{n=1}^{N} \frac{\partial}{\partial \lambda_{n}}[ & \lambda_{n}\left(1+\lambda_{n}\right) J_{\beta}\left(\left\{\lambda_{2}\right\}\right) \\
& \left.\times \frac{\partial}{\partial \lambda_{n}} \frac{w_{s}^{(\beta)}\left(\left\{\lambda_{\imath}\right\}\right)}{J_{\beta}\left(\left\{\lambda_{2}\right\}\right)}\right],
\end{aligned}
$$

where $J_{\beta}\left(\left\{\lambda_{\imath}\right\}\right) \equiv \prod_{n<m}\left|\lambda_{n}-\lambda_{m}\right|^{\beta}$. The initial condition is that of perfect transmission,

$$
w_{0}^{(\beta)}\left(\left\{\lambda_{2}\right\}\right)=\delta\left(\lambda_{1}\right) \delta\left(\lambda_{2}\right) \cdots \delta\left(\lambda_{N}\right) .
$$

The diffusion equation (3) is based on (a) the difference in symmetry properties of the ensemble of scattering matrices in the presence or absence of time-reversal symmetry; (b) an isotropy assumption, which implies that flux incident in one channel is, on average, equally distributed among all outgoing channels; and (c) a maximum entropy assumption for the distribution $w_{\delta s}^{(\beta)}\left(\left\{\lambda_{2}\right\}\right)$ for a small segment of the conductor. Assumption (b) requires a conductor much longer than wide, i.e., the quasi-onedimensional limit. Assumption (c) has been justified by a "central-limit theorem". ${ }^{16}$ Calculations for the conductance starting from Eq. (3) (Refs. 14 and 15) have indeed produced the same Ohmic conductance and quantuminterference effects (weak localization and UCF) as obtained earlier by Green's function techniques in the quasione-dimensional limit.

The outline of this paper is as follows. In Sec. II the diffusion equation (3) is used to determine the mesoscopic fluctuations in the shot-noise power. Furthermore, a weak-localization effect and the earlier found suppression by one-third are obtained for the ensemble-averaged shot-noise power. The calculation is straightforward, but lengthy. The key intermediate steps are given in the Appendix. Finally, the effect of inelastic scattering on the shot-noise power of conductors longer than the phasecoherence length is discussed in Sec. III.

\section{AVERAGE AND VARIANCE OF THE SHOT-NOISE POWER}

The regime of interest is the metallic, diffusive regime: The sample must be much longer than the mean free path, but much shorter than the one-dimensional 1(D) localization length $\xi \sim N \ell$, requiring

$$
1 \ll s \ll N \text {. }
$$

With the definition of the moment

$$
T_{q}^{r} \equiv\left(\sum_{n=1}^{N} \mathcal{T}_{n}^{q}\right)^{r} \equiv\left[\sum_{n=1}^{N} \frac{1}{\left(1+\lambda_{n}\right)^{q}}\right]^{r}
$$

and the convention $T^{p} \equiv T_{1}^{p}, T_{q} \equiv T_{q}^{1}$, and $T \equiv T_{1}^{1}$, one finds from Eq. (1) for the average and the variance of the shot-noise power the expressions

$$
\begin{aligned}
\left(2 e|V| 2 e^{2} / h\right)^{-1}\langle P\rangle= & \langle T\rangle-\left\langle T_{2}\right\rangle \\
\left(2 e|V| 2 e^{2} / h\right)^{-2} \operatorname{var} P= & \left\langle T^{2}\right\rangle-\langle T\rangle^{2} \\
& -2\left(\left\langle T T_{2}\right\rangle-\langle T\rangle\left\langle T_{2}\right\rangle\right) \\
& +\left\langle T_{2}^{2}\right\rangle-\left\langle T_{2}\right\rangle^{2} .
\end{aligned}
$$

The brackets denote the ensemble average,

$$
\langle F\rangle=\int_{0}^{\infty} d \lambda_{1} \int_{0}^{\infty} d \lambda_{2} \cdots \int_{0}^{\infty} d \lambda_{N} w_{s}^{(\beta)}\left(\left\{\lambda_{\imath}\right\}\right) F\left(\left\{\lambda_{\imath}\right\}\right) .
$$

From the diffusion equation (3) one can derive the evolution of the different moments. For example, the evolution equation for $\left\langle T^{p}\right\rangle$ is given by ${ }^{15}$

$$
\begin{aligned}
(\beta N+2-\beta) \frac{\partial}{\partial s}\left\langle T^{p}\right\rangle=\langle & -\beta p T^{p+1} \\
& -(2-\beta) p T^{p-1} T_{2} \\
& \left.+2 p(p-1) T^{p-2}\left(T_{2}-T_{3}\right)\right\rangle .
\end{aligned}
$$

Obviously, this single evolution equation is not solvable because of the appearance of additional moments. In Refs. 14 and 15 it is shown that the hierarchy of evolution equations can be closed by an expansion in powers of $N^{-1}$. The resulting set of coupled differential equations needed for the evaluation of Eqs. (7) and (8), and their solutions, are given in the Appendix. Here, only the results are presented.

For the average shot-noise power we find

$$
\langle P\rangle=2 e|V| \frac{2 e^{2}}{h}\left[\frac{N \ell}{3 L}-\frac{\delta_{\beta 1}}{45}+\mathcal{O}\left(\frac{L}{N \ell}\right)\right] .
$$

Combining this with the result for the average conductance from Ref. 15,

$$
\langle G\rangle=\frac{2 e^{2}}{h}\left[\frac{N \ell}{L}-\frac{\delta_{\beta 1}}{3}+\mathcal{O}\left(\frac{L}{N \ell}\right)\right]
$$

one can write

$$
\langle P\rangle=\frac{1}{3} P_{\text {Poisson }}+\delta P_{\mathrm{WL}},
$$

where $P_{\text {Poisson }} \equiv 2 e|V|\langle G\rangle$ and $\delta P_{\mathrm{WL}} \equiv\left(2 e|V| 2 e^{2} / h\right)$ $\times\left(4 \delta_{\beta 1} / 45\right)$. The suppression by a factor one-third of the ensemble-averaged Poisson noise is in agreement with Ref. 3, where the alternative global approach to randommatrix theory was used. In the second term of Eq. (11) one recognizes a weak-localization correction for the shot noise, analogous to that in Eq. (12) for the conductance. ${ }^{15}$ As it is caused by the interference between time-reversed pairs of trajectories, it disappears when time-reversal symmetry is broken $(\beta=2)$, i.e., in the presence of a magnetic field. The decrease in the conductance due to weak localization can be incorporated in the Poisson value. The remaining correction $\delta P_{W L}$ is positive, indicating that weak localization suppresses the conductance more than the shot noise.

Next the variance of the shot-noise power is determined. We find that the first two terms in the expansion of the right-hand-side of Eq. (8) - of order $N^{2}$ and $N$ respectively - vanish exactly. The remaining term is 


$$
\operatorname{var}\left(T-T_{2}\right)=\frac{1}{\beta} \frac{46}{2835}+\mathcal{O}\left(\frac{L}{N \ell}\right) .
$$

Thus, the root-mean-square fluctuations in the shot-noise power are given by

$$
\mathrm{rms} P=2 e|V| \frac{2 e^{2}}{h} C_{\beta},
$$

independent of the length $L$, the number of channels $N$, and the elastic mean free path $\ell$. By analogy with the conductance, one could speak of "universal noise fluctuations." The numerical coefficient is $C_{1}=\sqrt{46 / 2835} \simeq$ 0.127 in the presence of time-reversal symmetry and $C_{2}=\sqrt{23 / 2835} \simeq 0.090$ in its absence.

\section{EFFECT OF INELASTIC SCATTERING}

The theory presented is valid at zero temperature, when shot noise is the only source of current fluctuations and when all scattering is elastic. At finite temperatures the theory should be modified to include thermal noise (important when $k \mathrm{~T}>\mathrm{eV}$ ), the effects of thermal averaging [important when $L>l_{\mathrm{T}} \equiv(\hbar D / k \mathrm{~T})^{1 / 2}$, with $D$ the diffusion constant], and inelastic scattering (important when $L$ is greater than the phase-coherence length $\left.l_{\phi}\right)$. If $k \mathrm{~T} \ll e V$ and $l_{\phi} \ll l_{\mathrm{T}}$ the effect of inelastic scattering dominates. Its effect on the shot noise can be estimated by considering a model in which the conductor is divided into $M_{\phi} \simeq L / l_{\phi}$ phase-coherent segments of length $l_{\phi}$, separated by phase and momentum random- izing reservoirs. ${ }^{3}$ Quasi-one-dimensionality now requires that the width of the conductor be much smaller than $l_{\phi}$. Furthermore, phase-coherent diffusive transport requires $\ell \ll l_{\phi}$. In Ref. 3 the following sum rule was derived:

$$
R^{2} P=\sum_{\imath=1}^{M_{\phi}} R_{\imath}^{2} P_{\imath}
$$

where $R_{\imath}$ and $P_{\imath}$ are the resistance and the shot-noise power of an individual segment, and $R \equiv \sum_{\imath=1}^{M_{\phi}} R_{\imath}$ and $P$ are the resistance and the shot-noise power of the whole conductor. Using Eqs. (1) and (2), $R_{\imath}$ and $P_{\imath}$ can be expressed in terms of the moments $T(i)$ and $T_{2}(i)$ of the transmission eigenvalues of the $i$ th segment. Since a fraction $R_{2} / R$ of the total applied voltage $V$ drops over the $i$ th segment, one has from Eq. (16)

$$
P=2 e|V| \frac{2 e^{2}}{h} \frac{\sum_{\imath=1}^{M_{\phi}} T(i)^{-3}\left[T(i)-T_{2}(\imath)\right]}{\left[\sum_{\imath=1}^{M_{\phi}} T(\imath)^{-1}\right]^{3}} .
$$

Each moment in Eq. (17) can be written as the ensemble average plus a deviation, $T(i) \equiv\langle T\rangle_{\phi}+\delta T(\imath)$ and $T_{2}(\imath) \equiv\left\langle T_{2}\right\rangle_{\phi}+\delta T_{2}(i)$. The brackets $\langle\cdots\rangle_{\phi}$ denote the ensemble average for a phase-coherent conductor of length $l_{\phi}$. (All segments are assumed to have the same average properties.) Now Eq. (17) is expanded in powers of $\delta T(i)$ and $\delta T_{2}(i)$, and the fact that moments of different segments are statistically independent [e.g., $\langle\delta T(i) \delta T(j)\rangle=\langle\delta T(i)\rangle\langle\delta T(j)\rangle$, if $i \neq j]$ is used.

The ensemble-averaged shot-noise power becomes

$$
\langle P\rangle=2 e|V| \frac{2 e^{2}}{h} M_{\phi}^{-2}\left[\langle T\rangle_{\phi}-\left\langle T_{2}\right\rangle_{\phi}+3 \frac{M_{\phi}-1}{M_{\phi}}\left(\frac{\left\langle\delta T \delta T_{2}\right\rangle_{\phi}}{\langle T\rangle_{\phi}}-\frac{\left\langle(\delta T)^{2}\right\rangle_{\phi}\left\langle T_{2}\right\rangle_{\phi}}{\langle T\rangle_{\phi}{ }^{2}}\right)+\cdots\right]
$$

To determine the ensemble averages over a phasecoherent segment the results of Sec. II can be used (with $L$ substituted by $\left.l_{\phi}\right)$. One has $\langle T\rangle_{\phi},\left\langle T_{2}\right\rangle_{\phi}=\mathcal{O}\left(N \ell / l_{\phi}\right)$, while $\left\langle(\delta T)^{2}\right\rangle_{\phi},\left\langle\delta T \delta T_{2}\right\rangle_{\phi}=\mathcal{O}(1)$. It follows that the terms $\langle T\rangle_{\phi}$ and $\left\langle T_{2}\right\rangle_{\phi}$ in Eq. (18) are two orders of magnitude in $\left(N \ell / l_{\phi}\right)$ higher than the terms containing the fluctuations $\delta T$ and $\delta T_{2}$, so that it is consistent to neglect these latter terms while retaining the weak-localization corrections to $\langle T\rangle_{\phi}$ and $\left\langle T_{2}\right\rangle_{\phi}$. Equation (11) then implies

$$
\langle P\rangle=2 e|V| \frac{2 e^{2}}{h}\left[\frac{N \ell}{3 L}\left(\frac{l_{\phi}}{L}\right)-\frac{\delta_{\beta 1}}{45}\left(\frac{l_{\phi}}{L}\right)^{2}\right] .
$$

Comparison with Eq. (11) shows that, while the leading term in the average shot-noise power is reduced by a factor $\left(l_{\phi} / L\right)$ because of inelastic scattering, ${ }^{3}$ the weaklocalization correction is suppressed more strongly, by a factor $\left(l_{\phi} / L\right)^{2}$.

Now for the effect of inelastic scattering on the mesoscopic fluctuations of the shot-noise power. The variance $\left\langle P^{2}\right\rangle-\langle P\rangle^{2}$ is determined by substitution of the expres- sion for $P$ given in Eq. (17), then an expansion in powers of $\delta T$ and $\delta T_{2}$, and finally taking the ensemble average. The result is

$$
\begin{aligned}
\operatorname{var} P=\left(2 e|V| \frac{2 e^{2}}{h}\right)^{2} M_{\phi}^{-5}[ & \left\langle(\delta T)^{2}\right\rangle_{\phi}-2\left\langle\delta T \delta T_{2}\right\rangle_{\phi} \\
& \left.+\left\langle\left(\delta T_{2}\right)^{2}\right\rangle_{\phi}+\cdots\right] .
\end{aligned}
$$

The three terms between square brackets are, in fact, equal to the variance, $\operatorname{var}\left(T-T_{2}\right)$, of a phase-coherent segment of length $l_{\phi}$. With Eqs. (14) and (15) one finds

$$
\operatorname{rms} P=2 e|V| \frac{2 e^{2}}{h} C_{\beta}\left(\frac{l_{\phi}}{L}\right)^{5 / 2} \text {. }
$$

The root-mean-square value of the mesoscopic fluctuations of the shot-noise power is suppressed by a factor $\left(l_{\phi} / L\right)^{5 / 2}$ due to inelastic scattering. Hence, at the breakdown of phase-coherent transport the mesoscopic fluctuations cease being universal and become dependent on the length of the conductor.

The division of the conductor in phase-coherent seg- 
ments separated by phase-randomizing reservoirs is a simplified model of inelastic scattering, which occurs throughout the conductor. A more realistic treatment is expected to leave the parametric dependence on the ratio $\left(l_{\phi} / L\right)$ unaffected. It is interesting to compare the above results with the corresponding results for the conductance, ${ }^{17}$

$$
\begin{aligned}
& \delta G_{\mathrm{WL}}=\text { const } \times \frac{2 e^{2}}{h}\left(\frac{l_{\phi}}{L}\right), \\
& \mathrm{rms} G=\text { const } \times \frac{2 e^{2}}{h}\left(\frac{l_{\phi}}{L}\right)^{3 / 2} .
\end{aligned}
$$

\section{APPENDIX: MOMENT EXPANSION AND SOLUTION}

Consider a compound moment

$$
\prod_{\imath=1}^{m} T_{q_{2}}^{r_{2}} \equiv\left[\sum_{n=1}^{N} \frac{1}{\left(1+\lambda_{n}\right)^{q_{1}}}\right]^{r_{1}}\left[\sum_{n=1}^{N} \frac{1}{\left(1+\lambda_{n}\right)^{q_{2}}}\right]^{r_{2}} \ldots\left[\sum_{n=1}^{N} \frac{1}{\left(1+\lambda_{n}\right)^{q_{m}}}\right]^{r_{m}},
$$

where $q_{\imath} \neq q_{\jmath}$ if $\imath \neq \jmath$. From Eqs. (4) and (9) the initial condition of the ensemble average is

$$
\lim _{s \rightarrow 0}\left\langle\prod_{\imath} T_{q_{i}}^{r_{i}}\right\rangle=N^{\nu}, \nu \equiv \sum_{i=1}^{m} r_{\imath}
$$

The evolution of a compound moment can be derived from the diffusion equation (3). This leads to the general evolution equation

$$
\begin{aligned}
& (\beta N+2-\beta) \frac{\partial}{\partial s}\left\langle\prod_{\imath} T_{q_{2}}^{r_{2}}\right\rangle=\beta \sum_{\imath}\left\langle q_{\imath} r_{\imath} T_{q_{2}}^{r_{2}-1}\left(\sum_{n=0}^{q_{2}-2} T_{n+1} T_{q_{2}-n-1}-\sum_{n=0}^{q_{2}-1} T_{n+1} T_{q_{2}-n}\right) \prod_{\jmath \neq \imath} T_{q_{j}}^{r_{\jmath}}\right\rangle \\
& +(2-\beta) \sum_{\imath}\left\langle\left[q_{\imath}\left(q_{\imath}-1\right) r_{\imath} T_{q_{\imath}}^{r_{\imath}}-q_{\imath}{ }^{2} r_{\imath} T_{q_{\imath}}^{r_{2}-1} T_{q_{\imath}+1}\right] \prod_{\jmath \neq \imath} T_{q_{\jmath}}^{r_{\jmath}}\right\rangle \\
& +\sum_{\imath}\left\langle 2 q_{\imath}^{2} r_{\imath}\left(r_{\imath}-1\right) T_{q_{\imath}}^{r_{\imath}-2}\left(T_{2 q_{\imath}}-T_{2 q_{2}+1}\right) \prod_{\jmath \neq \imath} T_{q_{\jmath}}^{r_{\jmath}}\right\rangle \\
& +\sum_{\imath<\jmath}\left\langle 4 q_{\imath} q_{\jmath} r_{\imath} r_{\jmath} T_{q_{\imath}}^{r_{2}-1} T_{q_{\jmath}}^{r_{j}-1}\left(T_{q_{\imath}+q_{\jmath}}-T_{q_{2}+q_{\jmath}+1}\right) \prod_{k \neq \imath, j} T_{q_{k}}^{r_{k}}\right\rangle
\end{aligned}
$$

where $\sum_{n=a}^{b} \equiv 0$ if $a>b$. Equation (10) is a special case of Eq. (A3). The moments required for the average and the variance of the shot-noise power are $\langle T\rangle,\left\langle T_{2}\right\rangle,\left\langle T^{2}\right\rangle,\left\langle T T_{2}\right\rangle$, and $\left\langle T_{2}^{2}\right\rangle$, as can be seen from Eqs. (7) and (8). However, their evolution equations are not exactly solvable, because these cannot be written in a closed form. Mello and Stone $\mathrm{e}^{14,15}$ have developed a method of solution by expanding the moments in descending powers of $N$. Here, their general method is applied to the moments listed above. For this purpose the following expansions are necessary:

$$
\begin{aligned}
\left\langle T^{p}\right\rangle & =N^{p} f_{p, 0}(s)+N^{p-1} f_{p, 1}(s)+N^{p-2} f_{p, 2}(s)+\cdots, \\
\left\langle T^{p} T_{2}\right\rangle & =N^{p+1} g_{p+1,0}(s)+N^{p} g_{p+1,1}(s)+N^{p-1} g_{p+1,2}(s)+\cdots, \\
\left\langle T^{p} T_{3}\right\rangle & =N^{p+1} h_{p+1,0}(s)+N^{p} h_{p+1,1}(s)+\cdots, \\
\left\langle T^{p} T_{4}\right\rangle & =N^{p+1} \imath_{p+1,0}(s)+\cdots, \\
\left\langle T^{p} T_{5}\right\rangle & =N^{p+1} \jmath_{p+1,0}(s)+\cdots,
\end{aligned}
$$




$$
\begin{aligned}
\left\langle T^{p} T_{2}^{2}\right\rangle & =N^{p+2} l_{p+2,0}(s)+N^{p+1} l_{p+2,1}(s)+N^{p} l_{p+2,2}(s)+\cdots \\
\left\langle T^{p} T_{2} T_{3}\right\rangle & =N^{p+2} m_{p+2,0}(s)+N^{p+1} m_{p+2,1}(s)+\cdots \\
\left\langle T^{p} T_{2} T_{4}\right\rangle & =N^{p+2} n_{p+2,0}(s)+\cdots \\
\left\langle T^{p} T_{3}^{2}\right\rangle & =N^{p+2} o_{p+2,0}(s)+\cdots \\
\left\langle T^{p} T_{2}^{3}\right\rangle & =N^{p+3} t_{p+3,0}(s)+N^{p+2} t_{p+3,1}(s)+\cdots \\
\left\langle T^{p} T_{2}^{4}\right\rangle & =N^{p+4} u_{p+4,0}(s)+\cdots \\
\left\langle T^{p} T_{2}^{2} T_{3}\right\rangle & =N^{p+3} v_{p+3,0}(s)+\cdots
\end{aligned}
$$

The dependence of the coefficients of the powers of $N$ on the symmetry parameter $\beta$ is not explicitly mentioned. In this notation the expressions for the average (7) and the variance (8) of the shot noise can be obtained from

$$
\begin{aligned}
\left\langle T-T_{2}\right\rangle= & {\left[f_{1,0}(s)-g_{1,0}(s)\right] N+\left[f_{1,1}(s)-g_{1,1}(s)\right]+\left[f_{1,2}(s)-g_{1,2}(s)\right] N^{-1}+\cdots, } \\
\left\langle T^{2}\right\rangle-\langle T\rangle^{2}= & {\left[f_{2,0}(s)-f_{1,0}(s)^{2}\right] N^{2}+\left[f_{2,1}(s)-2 f_{1,0}(s) f_{1,1}(s)\right] N } \\
& +\left[f_{2,2}(s)-2 f_{1,0}(s) f_{1,2}(s)-f_{1,1}(s)^{2}\right]+\cdots, \\
\left\langle T T_{2}\right\rangle-\langle T\rangle\left\langle T_{2}\right\rangle= & {\left[g_{2,0}(s)-f_{1,0}(s) g_{1,0}(s)\right] N^{2}+\left[g_{2,1}(s)-f_{1,0}(s) g_{1,1}(s)-f_{1,1}(s) g_{1,0}(s)\right] N } \\
& +\left[g_{2,2}(s)-f_{1,0}(s) g_{1,2}(s)-f_{1,1}(s) g_{1,1}(s)-f_{1,2}(s) g_{1,0}(s)\right]+\cdots, \\
\left\langle T_{2}^{2}\right\rangle-\left\langle T_{2}\right\rangle^{2}= & {\left[l_{2,0}(s)-g_{1,0}(s)^{2}\right] N^{2}+\left[l_{2,1}(s)-2 g_{1,0}(s) g_{1,1}(s)\right] N } \\
& +\left[l_{2,2}(s)-2 g_{1,0}(s) g_{1,2}(s)-g_{1,1}(s)^{2}\right]+\cdots
\end{aligned}
$$

In order to determine the functions of $s$ listed above, the evolution equations of the moments of interest are set up from the generalized evolution equation (A3). One then finds that indeed all the moments of Eqs. (A4a)-(A4l) appear. Filling in the expansions and equating the coefficients of the same powers of $N$ leads to a closed hierarchy of recurrent differential equations:

$$
\begin{aligned}
& f_{p, 0}^{\prime}(s)+p f_{p+1,0}(s)=0 \\
& g_{p, 0}^{\prime}(s)+(p+3) g_{p+1,0}(s)=2 f_{p+1,0}(s) \\
& f_{p, 1}^{\prime}(s)+p f_{p+1,1}(s)=-\delta_{\beta 1}\left[f_{p, 0}^{\prime}(s)+p g_{p, 0}(s)\right] \\
& l_{p, 0}^{\prime}(s)+(p+6) l_{p+1,0}(s)=4 g_{p+1,0}(s) \\
& h_{p, 0}^{\prime}(s)+(p+5) h_{p+1,0}(s)=6 g_{p+1,0}(s)-3 l_{p+1,0}(s) \\
& g_{p, 1}^{\prime}(s)+(p+3) g_{p+1,1}(s)=2 f_{p+1,1}(s)+\delta_{\beta 1}\left[-g_{p, 0}^{\prime}(s)+2 g_{p, 0}(s)-4 h_{p, 0}(s)-(p-1) l_{p, 0}(s)\right] \\
& f_{p, 2}^{\prime}(s)+p f_{p+1,2}(s)=-\delta_{\beta 1}\left[f_{p, 1}^{\prime}(s)+p g_{p, 1}(s)\right]+\left(\delta_{\beta 1}+1\right) p(p-1)\left[g_{p-1,0}(s)-h_{p-1,0}(s)\right] \\
& t_{p, 0}^{\prime}(s)+(p+9) t_{p+1,0}(s)=6 l_{p+1,0}(s) \\
& m_{p, 0}^{\prime}(s)+(p+8) m_{p+1,0}(s)=2 h_{p+1,0}(s)+6 l_{p+1,0}(s)-3 t_{p+1,0}(s) \\
& l_{p, 1}^{\prime}(s)+(p+6) l_{p+1,1}(s)=4 g_{p+1,1}(s)+\delta_{\beta 1}\left[-l_{p, 0}^{\prime}(s)+4 l_{p, 0}(s)-8 m_{p, 0}(s)-(p-2) t_{p, 0}(s)\right] \\
& u_{p, 0}^{\prime}(s)+(p+12) u_{p+1,0}(s)=8 t_{p+1,0}(s) \\
& i_{p, 0}^{\prime}(s)+(p+7) i_{p+1,0}(s)=8 h_{p+1,0}(s)+4 l_{p+1,0}(s)-8 m_{p+1,0}(s) \\
& v_{p, 0}^{\prime}(s)+(p+11) v_{p+1,0}(s)=4 m_{p+1,0}(s)+6 t_{p+1,0}(s)-3 u_{p+1,0}(s) \\
& n_{p, 0}^{\prime}(s)+(p+10) n_{p+1,0}(s)=2 i_{p+1,0}(s)+8 m_{p+1,0}(s)+4 t_{p+1,0}(s)-8 v_{p+1,0}(s) \\
& o_{p, 0}^{\prime}(s)+(p+10) o_{p+1,0}(s)=12 m_{p+1,0}(s)-6 v_{p+1,0}(s) \\
& j_{p, 0}^{\prime}(s)+(p+9) j_{p+1,0}(s)=10 i_{p+1,0}(s)+10 m_{p+1,0}(s)-10 n_{p+1,0}(s)-5 o_{p+1,0}(s) \\
& t_{p, 1}^{\prime}(s)+(p+9) t_{p+1,1}(s)=6 l_{p+1,1}(s)+\delta_{\beta 1}\left[-t_{p, 0}^{\prime}(s)+6 t_{p, 0}(s)-12 v_{p, 0}(s)-(p-3) u_{p, 0}(s)\right] \\
& h_{p, 1}^{\prime}(s)+(p+5) h_{p+1,1}(s)=6 g_{p+1,1}(s)-3 l_{p+1,1}(s)+\delta_{\beta 1}\left[-h_{p, 0}^{\prime}(s)+6 h_{p, 0}(s)-9 i_{p, 0}(s)-(p-1) m_{p, 0}(s)\right] \\
& m_{p, 1}^{\prime}(s)+(p+8) m_{p+1,1}(s)=2 h_{p+1,1}(s)+6 l_{p+1,1}(s)-3 t_{p+1,1}(s) \\
& \quad+\delta_{\beta 1}\left[-m_{p, 0}^{\prime}(s)+8 m_{p, 0}(s)-4 o_{p, 0}(s)-9 n_{p, 0}(s)-(p-2) v_{p, 0}(s)\right] \\
&
\end{aligned}
$$




$$
\begin{aligned}
g_{p, 2}^{\prime}(s)+(p+3) g_{p+1,2}(s)= & 2 f_{p+1,2}(s)+\delta_{\beta 1}\left[-g_{p, 1}^{\prime}(s)+2 g_{p, 1}(s)-4 h_{p, 1}(s)-(p-1) l_{p, 1}(s)\right] \\
& +\left(\delta_{\beta 1}+1\right)\left\{4(p-1)\left[h_{p-1,0}(s)-i_{p-1,0}(s)\right]+(p-1)(p-2)\left[l_{p-1,0}(s)-m_{p-1,0}(s)\right]\right\}, \\
l_{p, 2}^{\prime}(s)+(p+6) l_{p+1,2}(s)= & 4 g_{p+1,2}(s)+\delta_{\beta 1}\left[-l_{p, 1}^{\prime}(s)+4 l_{p, 1}(s)-8 m_{p, 1}(s)-(p-2) t_{p, 1}(s)\right] \\
& +\left(\delta_{\beta 1}+1\right)\left\{8\left[i_{p-1,0}(s)-j_{p-1,0}(s)\right]+8(p-2)\left[m_{p-1,0}(s)-n_{p-1,0}(s)\right]\right. \\
& \left.+(p-2)(p-3)\left[t_{p-1,0}(s)-v_{p-1,0}(s)\right]\right\} .
\end{aligned}
$$

The equations are written in such order that each one can be solved with the solutions of the preceding ones. From Eq. (A2) the initial conditions are

$$
x_{p, 0}(0)=1, x_{p, 1}(0)=0, x_{p, 2}(0)=0
$$

where $x$ stands for each of the functions $f, g, h, \ldots, v$.

The first seven recurrent differential equations (A6a)$(\mathrm{A} 6 \mathrm{~g})$ were solved by Mello and Stone ${ }^{15}$ to determine the variance of the conductance. Guided by their results, the following ansatz for the solutions is made

$$
x_{p, \ldots}(s)=\frac{\pi(s)+p \sigma(s)+p^{2} \rho(s)}{(1+s)^{q}},
$$

where $\pi(s), \sigma(s)$, and $\rho(s)$ are functions in $s$ not dependent on $p$. The ansatz (A8) is then verified by substitution. In this way the recurrent differential equations (A6a)-(A6u) reduce, after an appropriate value for $q$ is chosen, to ordinary differential equations in $s$ for the functions $\pi(s), \sigma(s)$, and $\rho(s)$ which are easily solved. The functions are found to be polynomials in $s$. Here, only the solutions needed for substitution in Eqs. (A5a)(A5d) are presented:

$$
\begin{aligned}
& f_{p, 0}(s)=\frac{1}{(1+s)^{p}} \\
& f_{p, 1}(s)=\frac{-\delta_{\beta 1} p s^{3}}{3(1+s)^{p+2}} \\
& f_{p, 2}(s)=\frac{s^{2} p}{90(1+s)^{p+4}}\left\{\delta_{\beta 1}\left[(8 p-4) s^{4}+(18 p+6) s^{3}+(45 p+15) s^{2}+(60 p-60) s+45 p-45\right]\right. \\
& \left.+\left[(3 p-5) s^{4}+(18 p-30) s^{3}+(45 p-75) s^{2}+(60 p-90) s+45 p-45\right]\right\}, \\
& g_{p, 0}(s)=\frac{2 s^{3}+6 s^{2}+6 s+3}{3(1+s)^{p+3}} \\
& g_{p, 1}(s)=\frac{-\delta_{\beta 1} s^{3}}{45(1+s)^{p+5}}\left[(10 p+4) s^{3}+(30 p+24) s^{2}+(30 p+60) s+15 p+15\right], \\
& g_{p, 2}(s)=\frac{s^{2}}{1890(1+s)^{p+7}}\left\{\delta _ { \beta 1 } \left[\left(112 p^{2}+40 p-32\right) s^{7}+\left(588 p^{2}+612 p-120\right) s^{6}\right.\right. \\
& +\left(1722 p^{2}+2574 p+24\right) s^{5}+\left(3654 p^{2}+4326 p+924\right) s^{4} \\
& +\left(5418 p^{2}+2394 p+2772\right) s^{3}+\left(5355 p^{2}+315 p+5670\right) s^{2} \\
& \left.+\left(3150 p^{2}-630 p-2520\right) s+945 p^{2}+945 p-1890\right] \\
& +\left[\left(42 p^{2}-30 p-72\right) s^{7}+\left(378 p^{2}-270 p-648\right) s^{6}\right. \\
& +\left(1512 p^{2}-1080 p-2592\right) s^{5}+\left(3549 p^{2}-2457 p-5964\right) s^{4} \\
& +\left(5418 p^{2}-3402 p-8568\right) s^{3}+\left(5355 p^{2}-2835 p-8190\right) s^{2} \\
& \left.\left.+\left(3150 p^{2}-1260 p-5670\right) s+945 p^{2}+945 p-1890\right]\right\} \\
& l_{p, 0}(s)=\frac{1}{9(1+s)^{p+6}}\left(4 s^{6}+24 s^{5}+60 s^{4}+84 s^{3}+72 s^{2}+36 s+9\right), \\
& l_{p, 1}(s)=\frac{-\delta_{\beta 1} s^{3}}{135(1+s)^{p+8}}\left[(20 p+16) s^{6}+(120 p+144) s^{5}+(300 p+576) s^{4}+(420 p+1332) s^{3}\right. \\
& \left.+(360 p+1764) s^{2}+(180 p+1260) s+45 p+450\right],
\end{aligned}
$$




$$
\begin{aligned}
l_{p, 2}(s)=\frac{s^{2}}{28350(1+s)^{p+10}\left\{\delta_{\beta 1}\right.}[ & \left(1120 p^{2}+1360 p+4\right) s^{10}+\left(9240 p^{2}+17160 p+3408\right) s^{9} \\
& +\left(38220 p^{2}+91860 p+33864\right) s^{8}+\left(107520 p^{2}+273840 p+152480\right) s^{7} \\
& +\left(224280 p^{2}+497880 p+413820\right) s^{6}+\left(351540 p^{2}+596340 p+780120\right) s^{5} \\
& +\left(409500 p^{2}+506520 p+917280\right) s^{4}+\left(345870 p^{2}+334530 p+517860\right) s^{3} \\
& +\left(203175 p^{2}+212625 p+66150\right) s^{2}+\left(75600 p^{2}+113400 p-151200\right) s \\
& \left.+14175 p^{2}+42525 p-28350\right] \\
+ & \left(420 p^{2}+100 p-1020\right) s^{10}+\left(5040 p^{2}+1200 p-12240\right) s^{9} \\
& +\left(27720 p^{2}+6600 p-67320\right) s^{8}+\left(92820 p^{2}+22260 p-223840\right) s^{7} \\
& +\left(211680 p^{2}+51840 p-499860\right) s^{6}+\left(345240 p^{2}+88560 p-795240\right) s^{5} \\
& +\left(407925 p^{2}+114345 p-943110\right) s^{4}+\left(345870 p^{2}+134190 p-842940\right) s^{3} \\
& +\left(203175 p^{2}+146475 p-538650\right) s^{2}+\left(75600 p^{2}+103950 p-245700\right) s \\
+ & \left.\left.14175 p^{2}+42525 p-28350\right]\right\} .
\end{aligned}
$$

The solutions (A9a)-(A9e) and (A9g) have already been found by Mello and Stone. ${ }^{15}$ We have checked by computer algebra that the complete set of solutions indeed satisfies the set of recurrent differential equations (A6a)-(A6u) and the initial conditions (A7).

Using the solutions (A9a)-(A9f) one then obtains for Eq. (A5a)

$$
\left\langle T-T_{2}\right\rangle=\frac{s\left(s^{2}+3 s+3\right)}{3(1+s)^{4}} N-\frac{\delta_{\beta 1} s^{3}\left(s^{3}-9 s^{2}+\cdots\right)}{45(1+s)^{6}}-\frac{\left(2 \delta_{\beta 1}-1\right) s^{3}\left(3 s^{6}+27 s^{5}+\cdots\right)}{315(1+s)^{8}} N^{-1}+\cdots
$$

Equation (11) follows from Eq. (A10) by omitting terms of order $N s^{-2}, s^{-1}$, and $s N^{-1}$, while retaining terms of order $N s^{-1}$ and 1. This is a consistent approximation if $N^{1 / 2} \ll s \ll N$, which is a stronger condition than Eq. (5). As discussed in Ref. 15 this condition implies the quasi-one-dimensionality (length $\gg$ width) of the conductor. The variance can be calculated by filling in the solutions (A9a)-(A9i) in Eqs. (A5b)-(A5d). The terms of order $N^{2}$ and $N$ vanish and the remaining term is

$$
\operatorname{var}\left(T-T_{2}\right)=\frac{\left(1+\delta_{\beta 1}\right) s^{2}\left(23 s^{10}+276 s^{9}+1518 s^{8}+\cdots\right)}{2835(1+s)^{12}}+\cdots
$$

Taking the limit of a long system $(s \gg 1)$ results in Eq. (14).

${ }^{1}$ See, for example: R. Landauer and Th. Martin, Physica B 175, 167 (1991); M. Büttiker, ibid. 175, 199 (1991), and references therein.

${ }^{2}$ A. van der Ziel, Noise: Sources, Characterization, Measurement (Prentice-Hall, Englewood Cliffs, NJ, 1970).

${ }^{3}$ C.W.J. Beenakker and M. Büttiker, Phys. Rev. B 46, 1889 (1992).

${ }^{4} \mathrm{~A}$ review of mesoscopic fluctuation phenomena is Mesoscopic Phenomena in Solids, edited by B.L. Al'tshuler, P.A. Lee, and R.A. Webb (North-Holland, Amsterdam, 1991).

${ }^{5}$ B.L. Al'tshuler, Pis'ma Zh. Eksp. Teor. Fiz. 41, 530 (1985) [JETP Lett. 41, 648 (1985)].

${ }^{6}$ P.A. Lee and A.D. Stone, Phys. Rev. Lett. 55, 1622 (1985).

${ }^{7}$ M. Büttiker, Phys. Rev. Lett. 65, 2901 (1990).

${ }^{8}$ V.A. Khlus, Zh. Eksp. Teor. Fiz. 93, 2179 (1987) [Sov. Phys. - JETP 66, 1243 (1987)].
${ }^{9}$ G.B. Lesovik, Pis'ma Zh. Eksp. Teor. Fiz. 49, 513 (1989) [JETP Lett. 49, 592 (1989)].

${ }^{10}$ B. Yurke and G.P. Kochanski, Phys. Rev. B 41, 8184 (1990).

${ }^{11}$ Y. Imry, Europhys. Lett. 1, 249 (1986).

${ }^{12}$ K.A. Muttalib, J.-L. Pichard, and A.D. Stone, Phys. Rev. Lett. 59, 2475 (1987).

${ }^{13}$ P.A. Mello, P. Pereyra, and N. Kumar, Ann. Phys. 181, 290 (1988).

${ }^{14}$ P.A. Mello, Phys. Rev. Lett. 60, 1089 (1988).

${ }^{15}$ P.A. Mello and A.D. Stone, Phys. Rev. B 44, 3559 (1991).

${ }^{16}$ A.D. Stone, P.A. Mello, K.A. Muttalib, and J.-L. Pichard, Mesoscopic Phenomena in Solids (Ref. 4).

${ }^{17}$ P.A. Lee, A.D. Stone, and H. Fukuyama, Phys. Rev. B 35, 1039 (1987); C.W.J. Beenakker and H. van Houten, Solid State Phys. 44, 1 (1991). 